\title{
A Feature Extraction Algorithm for the Detection of "Five-Distances" in Hazardous Chemical Storage and Stacking in Night Vision Environment
}

\author{
Xuejun Liu ${ }^{1}$, Yuchen Wei ${ }^{1(\bowtie)}$, Jiandong Zhang ${ }^{1}$, Bo $\mathrm{Li}^{1}$, Jin Wang ${ }^{1}$, \\ Bo Dai ${ }^{1}$, and Cuiqing $\mathrm{Li}^{2}$ \\ ${ }^{1}$ College of Information Engineering, Beijing Institute of Petrochemical \\ Technology (BIPT), Beijing 102617, China \\ ${ }^{2}$ College of Chemistry Engineering, Beijing Institute of Petrochemical \\ Technology (BIPT), Beijing 102617, China
}

\begin{abstract}
The In recent years, safety distance monitoring of hazardous chemical stacking has become an increasingly important problem to be solved. It is a promising solution to build "Five-Distances" of binocular camera detection system based on cloud platform for intelligent safety distance warning. Aiming at the problem of feature extraction in "Five-Distances" detection of stacking in night vision environment, this paper proposes the construction of twodimensional OSTU average gray function window and the average algorithm of threshold data results. The threshold value of feature extraction is calculated by combining the probability density distribution of foreground and background. Experiments on standard data sets and simulated experimental scene images show that this algorithm significantly reduces the number of noise points and improves the accuracy of subsequent edge detection and corner point detection compared with the gray straight square homogenization algorithm.
\end{abstract}

Keywords: Night vision - Two-dimensional OSTU · Function window · Feature extraction - "Five-Distances" detection · Hazardous chemical stacking

\section{The Introduction}

In recent years, the safety accidents of hazardous chemical stacking happen frequently, and there were many casualties, Such as Tianjin port incident, Shenghua chemical of Hebei Zhangjiakou incident $[1,2]$. Therefore, more and more attention should be paid to the supervision of hazardous chemicals. However, the intelligent supervision measures are more important and effective scientific means in the new age [3]. At present, the research on safety Supervision and management of hazardous chemical stacking is developing gradually. There are laser scanning system, UWB positioning system and so on $[4,5]$. In this paper, binocular cameras are used to build a "Five-Distance" measuring platform for hazardous chemical stacking, and use cloud services as a communication platform to supervise. In the measurement of binocular "Five-Distances", it is divided into camera calibration, corner point matching, depth information and other steps. The picture is shown in Fig. 1. 


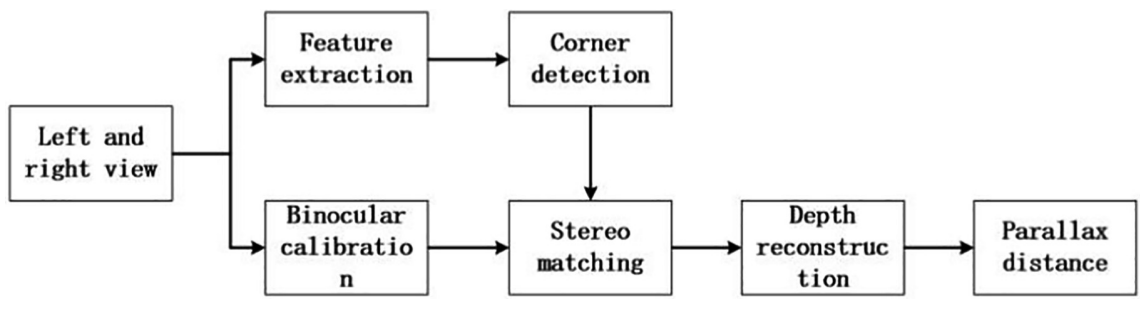

Fig. 1. Flow chart of binocular "Five-Distance" test system.

Feature extraction is related to the subsequent algorithm modules and greatly affects the accuracy of distance measurement results. Especially in the environment of night vision, pixel gray value is close to each other. In this paper, the two-dimensional OSTU average gray function window construction and threshold averaging algorithm are proposed to achieve the stacking feature extraction and corner point detection in the night vision environment, providing information for the subsequent "five distances" measurement.

\section{Feature Extraction Algorithm for Stacking in Night Vision Environment}

In the night vision environment, we use the binocular camera model 1920*1080 resolution. The configuration parameters of the computer are Inter Core i5-4200, 2G DDR3 independent graphics card, 8G memory and 500G hard disk. The pictures taken in the night vision environment are shown in Fig. 2.

Among them, the stacking was simulated with cube wood blocks. Figure 3 is the corner detection diagram of the image Fig. 2 in the night vision environment.

Therefore, binary ideas are selected to extract object information. However, the traditional one-dimensional OSTU cannot retrieve the information around the point, which may lead to inaccurate threshold and include noise [6]. The two-dimensional OSTU can avoid such problems. But construction of the average grayscale function window and two-dimensional threshold is always the core problem of this kind of algorithm [7, 8]. Therefore, this paper analyzes this problem and gives construction of the average grayscale function window and two-dimensional threshold averaging. After the foreground and background are extracted, Sobel algorithm is used to extract the edge of gradient dimensionality reduction, so as to carry out subsequent steps such as corner detection. Figure 4 shows the improved two-dimensional OSTU and edge extraction algorithm flow chart.

According to the steps in Fig. 4, the first step is to establish a two-dimensional gray scale array, where the first dimension is the gray value of a point, and the second 

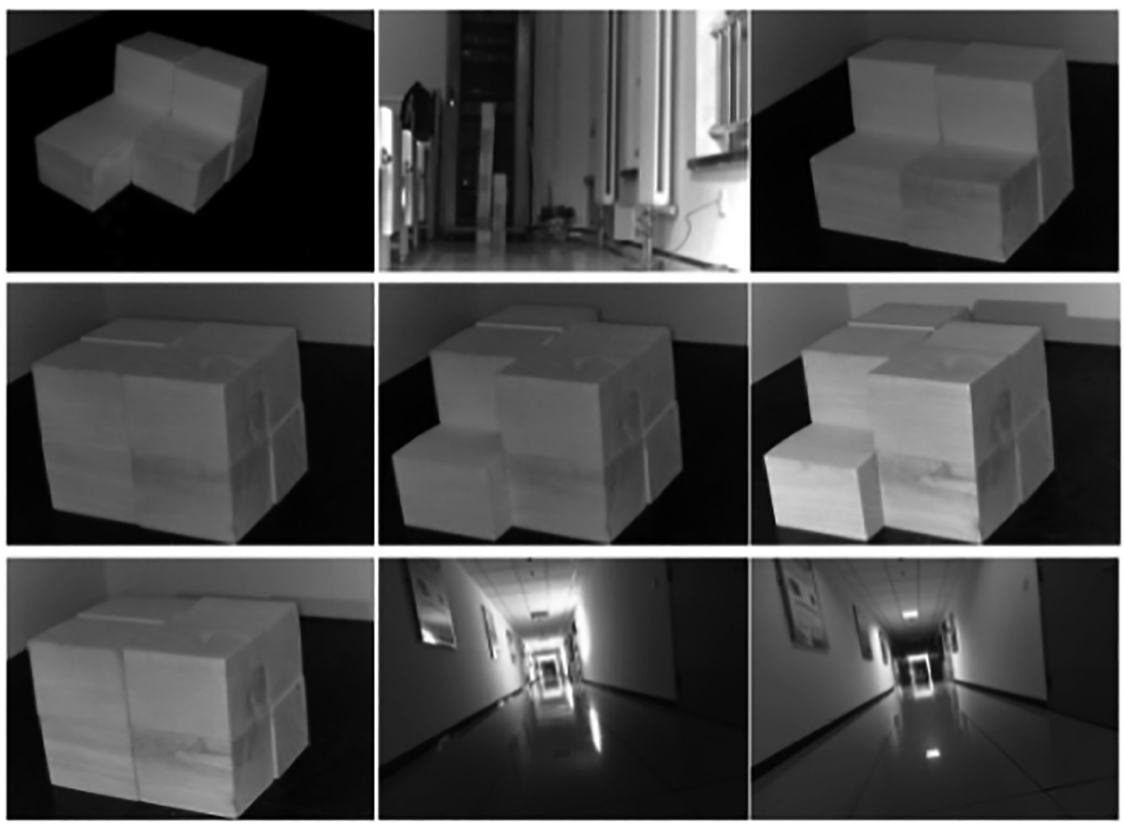

Fig. 2. The image of night vision environment.
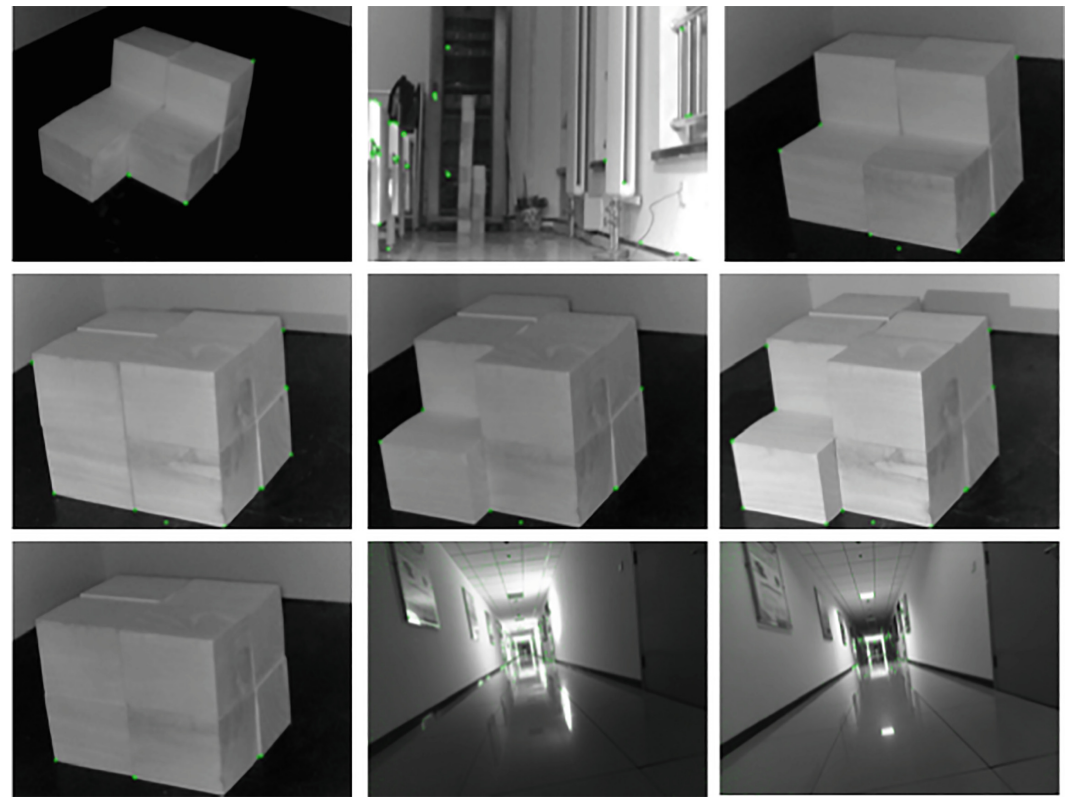

Fig. 3. The image of corner detection environment in the night vision 


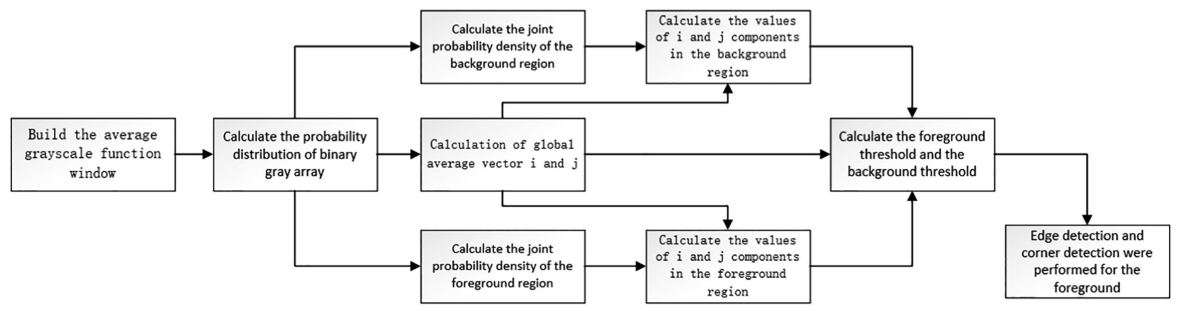

Fig. 4. Flow chart of improved two-dimensional OSTU and edge extraction algorithm

dimension data is centered on the point, and the average value of all points in a window is taken, as shown in formula (1).

$$
\left\{\begin{array}{c}
\left(\sum_{i=0}^{\text {height }} \sum_{j=0}^{\text {wide }} \sum_{m=i-1}^{i+1} \sum_{n=j-1}^{j+1} D_{m * T+n}^{\prime \prime}\right) /(x * y) \\
\sum_{i=0}^{\text {height }} \sum_{j=0}^{\text {width }} D_{i * T+j}^{\prime}
\end{array},[m \geq 0, m<\text { height }, n \geq 0, n<\text { width }]\right.
$$

In formula (1), I and $\mathrm{j}$ are iteration times respectively, Wide and height are the number of pixels in the image, $m$ and $n$ are the position of the length and width of the function window deviating from the pixel dot at a certain point, $\mathrm{T}$ is the actual memory length of the image, $x$ and $y$ are the length and width of the function window respectively, Its size is the value of the difference between the starting point and the ending point of $\mathrm{m}$ and $n$ respectively, $D_{m * T+n}^{\prime \prime}$ is the grayscale value of a point in the function window, $\mathrm{D}_{\mathrm{i} * \mathrm{~T}+\mathrm{j}}^{\prime}$ is the grayscale value of each pixel of the picture. After that, the joint probability distribution of this two-dimensional array is calculated [6,7]. As shown in formula (2).

$$
P_{i j}=\sum_{i=0}^{L} \sum_{j=0}^{L}\left(G_{i j} / \text { height } * \text { width }\right)
$$

In formula (2), $\mathrm{P}_{\mathrm{ij}}$ is the probability distribution, $\mathrm{L}$ is the maximum value of grayscale, its value is $256, \mathrm{G}_{\mathrm{ij}}$ is the number of occurrences of the same binary group. Then the joint probability density of the background region and the joint probability density of the foreground region are calculated respectively [6, 7]. As shown in formulas (3), (4).

$$
\begin{gathered}
\omega_{1}=\sum_{i=0}^{s} \sum_{j=0}^{t} P_{i j} \\
\omega_{2}=\sum_{i=s+1}^{L-1} \sum_{j=t+1}^{L-1} P_{i j}
\end{gathered}
$$

$\omega_{1}$ is the joint probability density of the foreground, $\omega_{2}$ is the joint probability density of the backgrounds, $s, t$ is the binary group threshold. The value of the foreground 
component $\mathrm{i}$ and $\mathrm{j}$, the value of the background component $\mathrm{i}$ and $\mathrm{j}$, the value of the average vector $i$ and $j$. It is respectively obtained from the following formulas (5), (6) and $(7)[6,7]$.

$$
\begin{gathered}
\mu_{1}=\left(\mu_{1 i}, \mu_{1 j}\right)^{T}=\left(\sum_{i=0}^{s} \sum_{j=0}^{t} \frac{i * P_{i j}}{\omega_{1}}, \sum_{i=0}^{s} \sum_{j=0}^{t} \frac{j * P_{i j}}{\omega_{1}}\right)^{T} \\
\mu_{2}=\left(\mu_{2 i}, \mu_{2 j}\right)^{T}=\left(\sum_{i=s+1}^{L-1} \sum_{j=t+1}^{L-1} \frac{i * P_{i j}}{\omega_{2}}, \sum_{i=0}^{s} \sum_{j=0}^{t} \frac{j * P_{i j}}{\omega_{2}}\right)^{T} \\
\mu_{T}=\left(\mu_{T i}, \mu_{T j}\right)^{T}=\left(\sum_{i=0}^{L-1} \sum_{j=0}^{L-1} i * P_{i j}, \sum_{i=0}^{L-1} \sum_{j=0}^{L-1} i * P_{i j}\right)^{T}
\end{gathered}
$$

$\mu_{1}$ is the values of the foreground component $i$ and $j$, Among them, $\mu_{1 i}$ the value of the foreground component $i, \mu_{1 j}$ is the value of the foreground component $j . \mu_{\mathrm{T}}$ are the values of global region components $i$ and $j$. Among them, $\mu_{\mathrm{Ti}}$ is the value of global region component $i$, and $\mu_{\mathrm{Tj}}$ is the value of global region component $j[6,7]$. The twodimensional maximum peak difference is shown in formula (8).

$$
\begin{aligned}
\operatorname{Max}\{\operatorname{TrMax}\}= & \operatorname{Max}\left\{\left[\left(\omega_{1} * \mu_{T i}-\mu_{1 i}\right) *\left(\omega_{1} * \mu_{T i}-\mu_{1 i}\right)+\left(\omega_{1} * \mu_{T j}-\mu_{1 j}\right)\right.\right. \\
& \left.\left.*\left(\omega_{1} * \mu_{T j}-\mu_{1 j}\right)\right] /\left(\omega_{1} * \omega_{2}\right)\right\}
\end{aligned}
$$

In formula (8), $\operatorname{Max}\{\operatorname{TrMax}\}$ is the maximum peak difference after iteration. The point where the maximum two-dimensional peak difference occurs is the demarcation point between foreground and background. But two dimensional points contain the values of $\mathrm{i}$ and $\mathrm{j}$. One is the current gray value of the demarcation point. The other one is the gray value of the window function of this gray point. The final binary demarcation point formula is shown in (9).

$$
\sqrt{\mathrm{i} * \mathrm{j}} / 1=(0.99999 \sim 1.00000)\left\{N \rightarrow\left(\begin{array}{c}
Y \rightarrow \mathrm{i} \\
+\mathrm{j}
\end{array}\right) / 2\right.
$$

In formula (10), I and $\mathrm{j}$ are respectively the position coordinates of the maximum peak difference $\operatorname{Max}\{\operatorname{TrMax}\}$. In other words, this is the binary gray threshold, If the binary group threshold is processed by $\sqrt{\mathrm{i} * \mathrm{j}} / \mathrm{i}$, its value is close to 1 , Then the threshold of the foreground and background is $i$, otherwise its value is $(i+j) / 2$, That's the average value of the binary group. 


\section{Analysis of Experimental Results}

According to the above two - dimensional OSTU window function setting algorithm and two - dimensional threshold averaging algorithm, The next step is to test the standard data set and the simulated experimental scene data set respectively.

\subsection{The Test Results of Standard Data Sets}

Figure 5 is screenshot of some randomly selected images from the standard data set downloaded from GitHub.

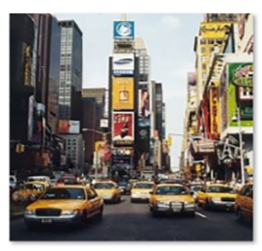

art11.jpg

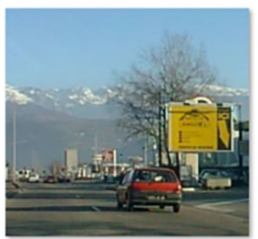

gre143.jpg

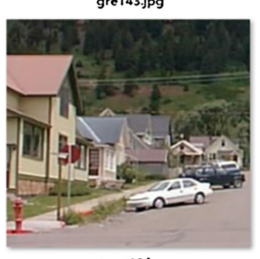

street18.jpg

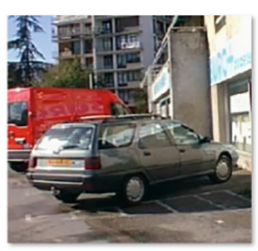

art665.jpg

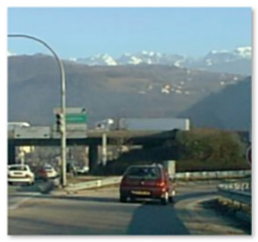

gre152.jpg

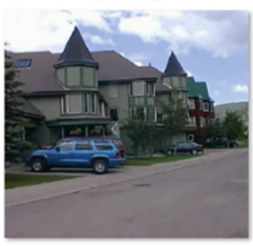

street115.jpg

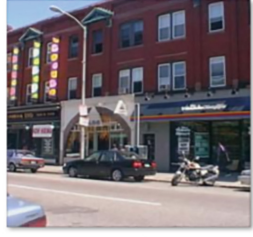

bost27.jpg

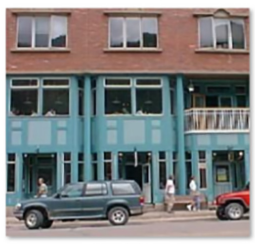

hous20.jpg

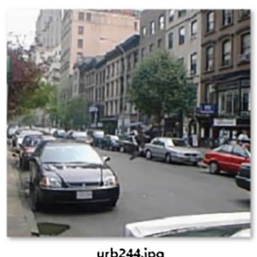

urb244.jpg

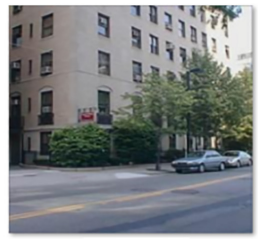

bost112.jpg

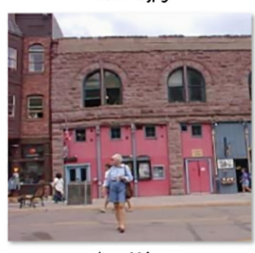

hous $30 . j p g$

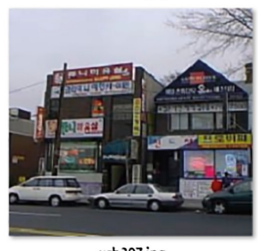

urb307.jpg

Fig. 5. The screenshots of some randomly selected images from the standard data set.

For the data set picture shown in Fig. 5, Construction of the average grayscale function window and the average of two-dimensional threshold were carried out. Figure 6 is the binary image corresponding to Fig. 5. 


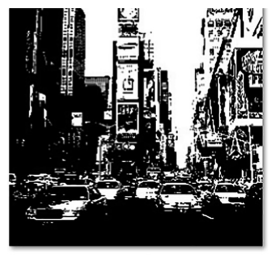

art11.jpg

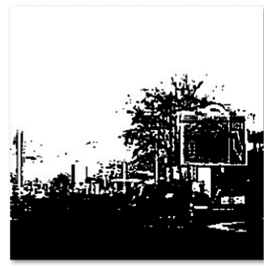

gre143.jpg

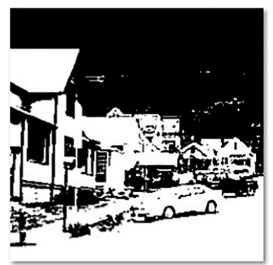

street18.jpg
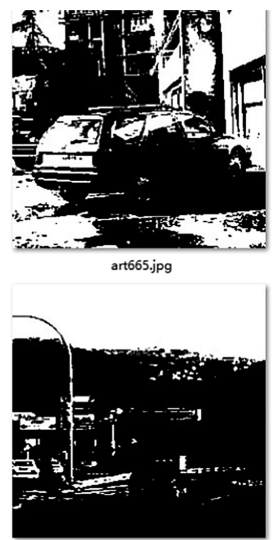

gre152.jpg

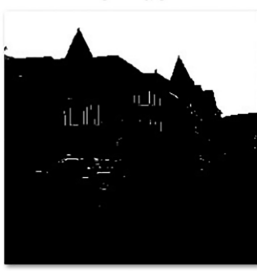

street115.jpg

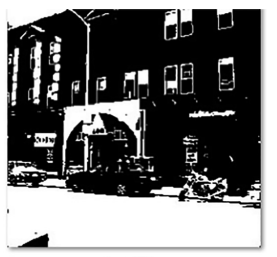

bost27.jpg

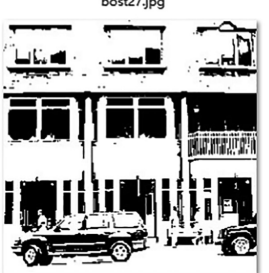

hous20.jpg

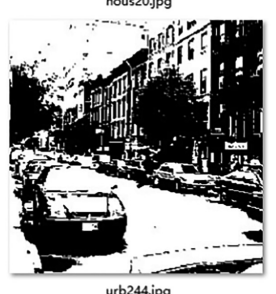

urb244.jpg
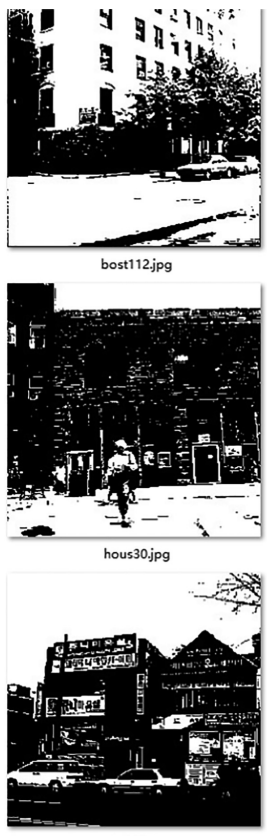

urb307.jpg

Fig. 6. Binary images corresponding to some images randomly selected from the standard data set.

According to whether the constructed grayscale distribution is on the negative diagonal, the data-oriented evaluation algorithm is excellent [7, 8]. As shown in Fig. 7, the gray distribution corresponding to Fig. 6 is on the negative diagonal.

As show in Fig. 7, the randomly selected standard data set distributed on the negative diagonal, only art11.png is abnormal, but the binarization effect in Fig. 6 is acceptable. 

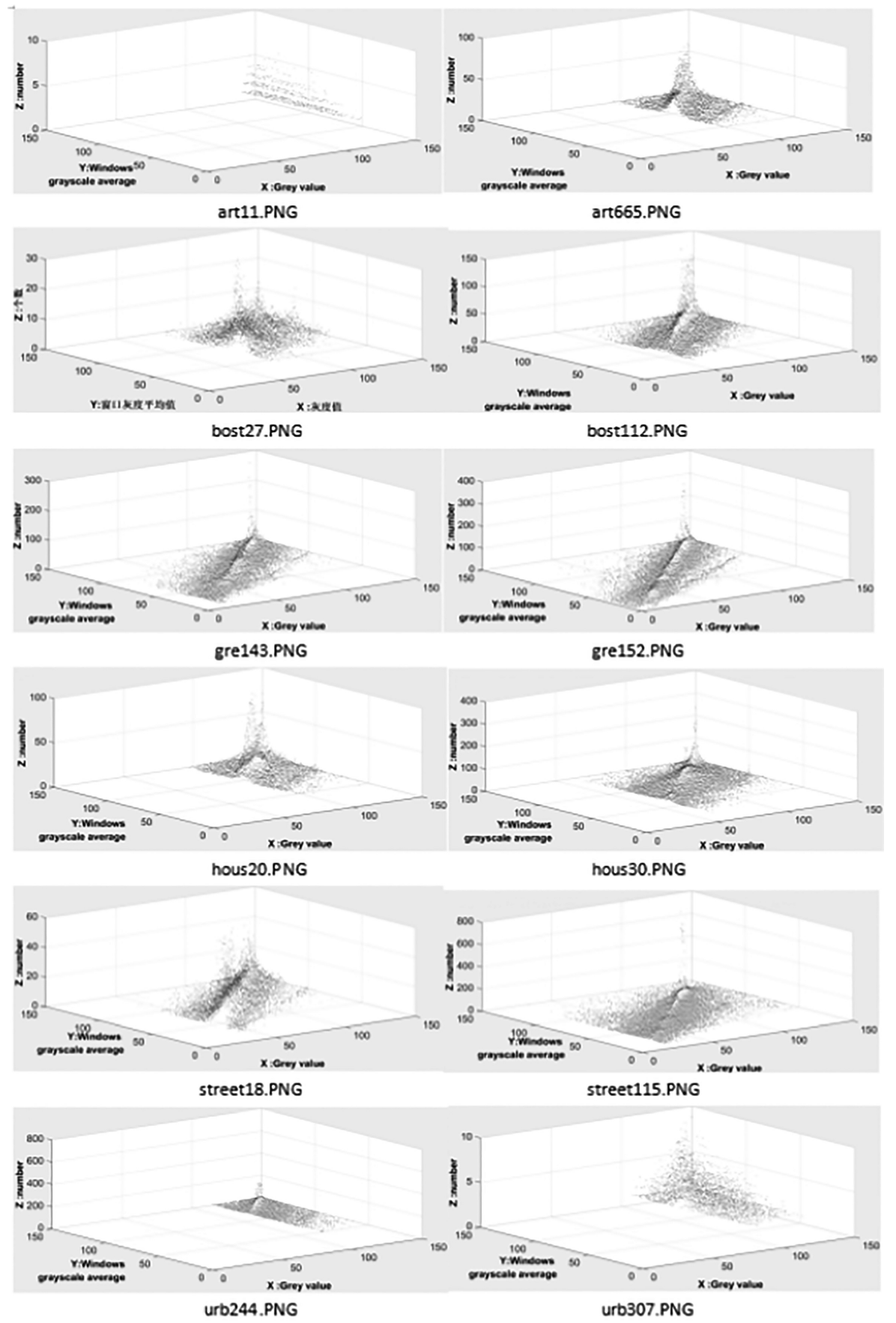

Fig. 7. The three-dimensional point distribution diagram corresponding to some images randomly selected from the standard data set. 


\subsection{Test Results of Simulated Experiment Scenes}

The experimental simulation scenes are full-stack (That is the maximum threedimensional standard size of the case, the critical point of the "Five -Distance" alarm), over-stack (That is beyond the maximum three-dimensional standard size of the case, the alarm state) and owe-stack (That is the three-dimensional size is less than the standard size of the case, the safe state). As shown in Fig. 8 .

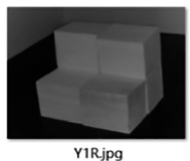

Y1R.jpg

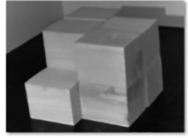

Y4R.jpg
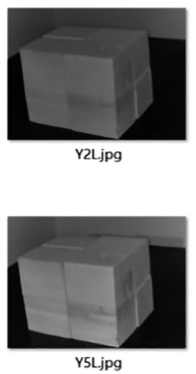
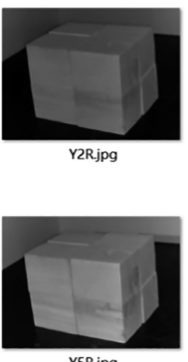

Y5R.jpg
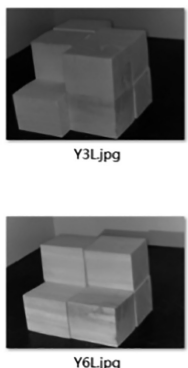

Y6L.jpg
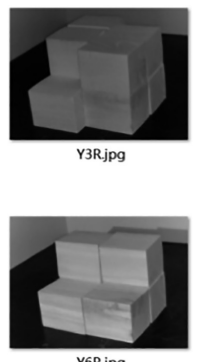

Y6R.jpg

Fig. 8. The experiment simulation scene graph of full-stack, over-stack, owe-stack.

Y1R.jpg, Y5L.jpg, Y5R.jpg in Fig. 8 are left and right views under different light and shadow in the case of the stack respectively. The $\mathrm{R}$ represents the left view and $\mathrm{L}$ represents the right view (the following picture naming rules are the same). Y21.jpg, Y2R.jpg, Y5L.jpg, Y5R.jpg are left and right views under different light and shadow in the case of full stack respectively. Y21.jpg, Y3L.jpg, Y3R.jpg, Y4R.jpg are left and right views under different light and shadow in the case of the over-stack respectively. The algorithm in this paper is applied to Figs. 8 and 9 shows the binary image processed in Fig. 8.
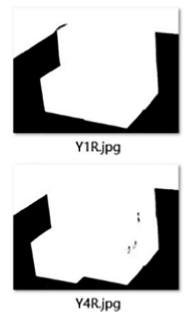
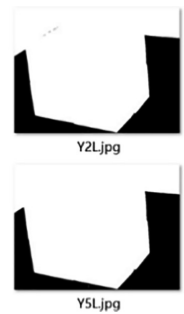
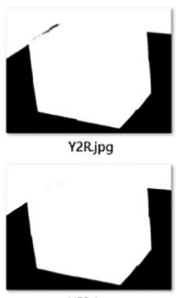

Y5R.jpg
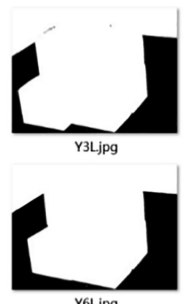

Y6L.jpg
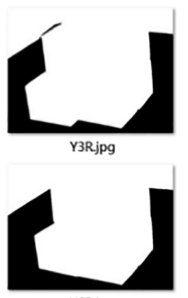

Y6R.jpg

Fig. 9. The binarization diagram of the simulation scenes of full-stack, over-stack and owe-stack.

Figure 9 is numerically analyzed according to whether the constructed grayscale distribution is on the negative diagonal [7,8]. As shown in Fig. 10, the gray scale distribution corresponding to Fig. 9 is on the negative diagonal. 


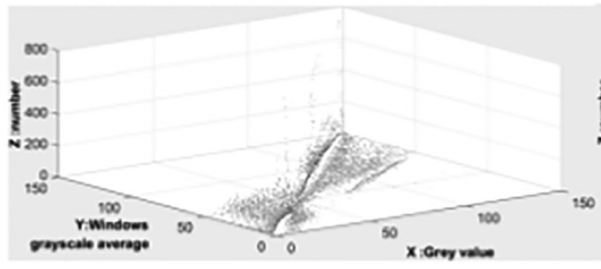

Y1R.PNG

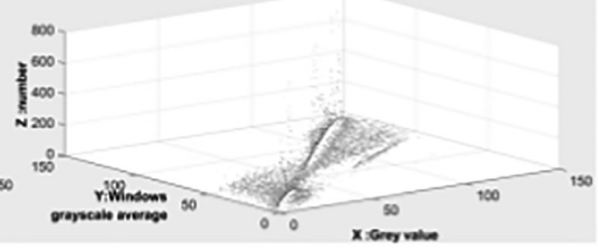

Y2L.PNG
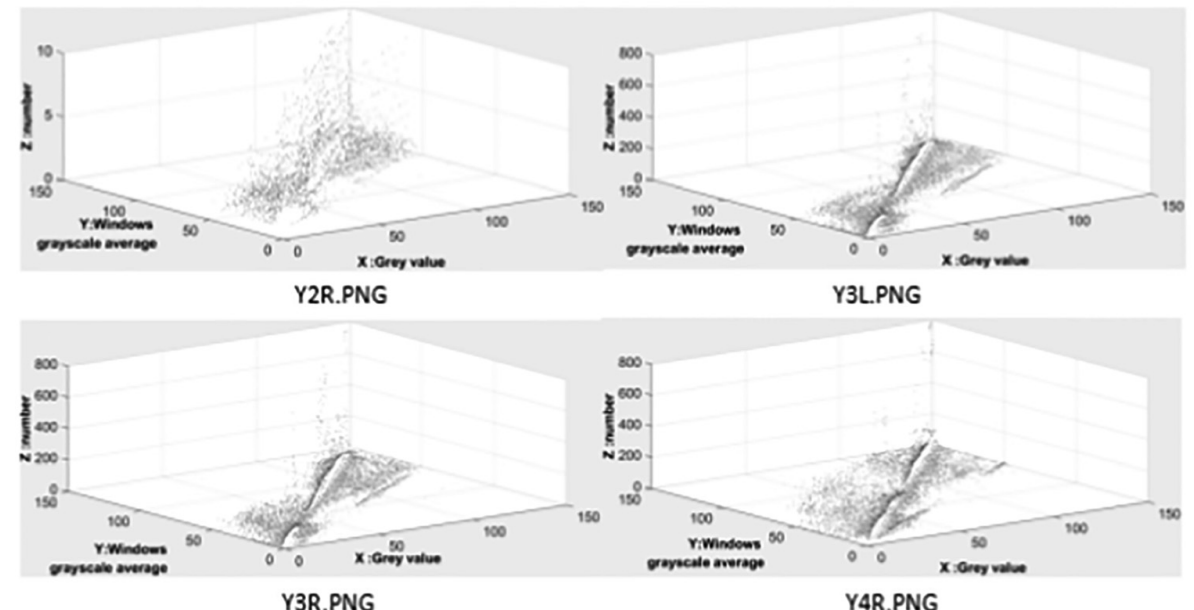

Y3R.PNG

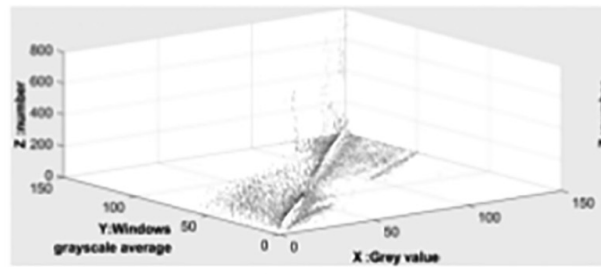

\section{Y5L.PNG}

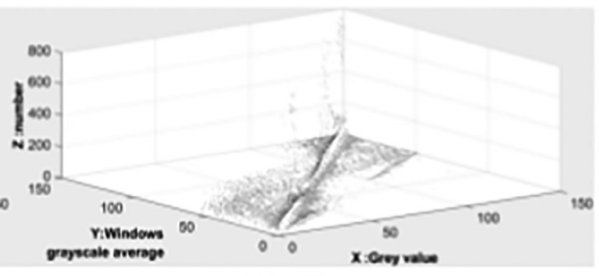

Y5R.PNG

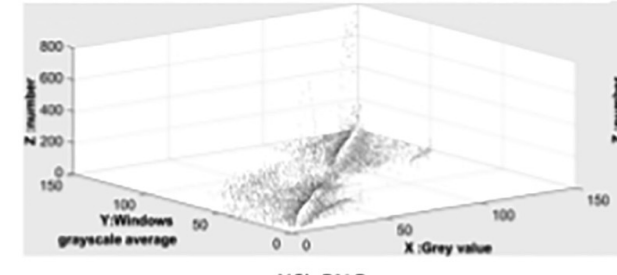

Y6L.PNG

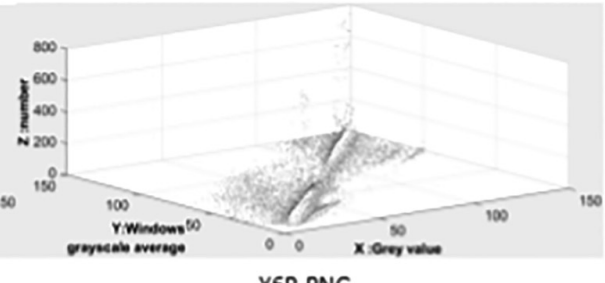

Y6R.PNG

Fig. 10. The three-dimensional point distribution diagram of the simulated scenes of full-stack, over-stack and owe-stack.

It can be seen from Fig. 10 that the binarization $3 \mathrm{~d}$ points in the experimental simulation scene can be roughly distributed on the negative diagonal to reach $100 \%$. 


\subsection{Edge Extraction and Corner Detection Were Used to Verify the Results}

Considering the low definition of Fig. 2 in the case of night vision, take measures to remove ambiguities, Gray histogram, one-dimensional OSTU and other algorithms were selected [9-11]. Edge detection and corner detection are performed and compared with the algorithm in this paper. As shown in Fig. 11, the original night vision picture, the night vision picture processed with gray level square homogenization, the picture used Sobel algorithm to extract the edge based on the left picture, the night vision picture that contain the corner position information are from left to right.

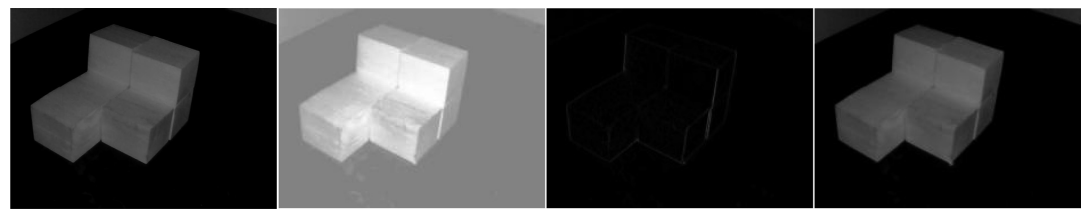

Fig. 11. Edge detection picture and corner detection picture after homogenization treatment.

As shown in Fig. 11, After the homogenization treatment of the gray level square of the experimental night view, the gray level value range produces a phenomenon of concentration, the edge is not relatively clear when extracting, and the corner points cannot be identified. From left to right, as shown in Fig. 12, is the original night view. The binomial picture after the one-dimensional OSTU based on the left picture, the pictures further using sobel algorithm for edge detection based on the left picture and the night vision picture that contain the corner position information.

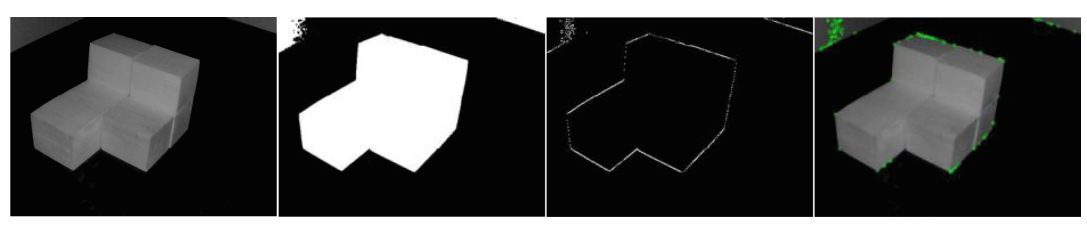

Fig. 12. Edge detection and corner detection pictures after one-dimensional OSTU processing.

As shown in Fig. 12, although the edge and corner information of the object can be detected after the one-dimensional OSTU processing, the binarization image does not effectively extract the foreground information, As a result, the original night view contains a lot of noise information, that is, the upper left corner of pictures 2, 3 and 4 in Fig. 12. As shown in Fig. 13, from left to right are the original night view, the binomial view after two-dimensional OSTU processing based on the original image, the edge detection diagram of sobel algorithm after two-dimensional OSTU processing, and the night view after sobel algorithm corner point detection. 


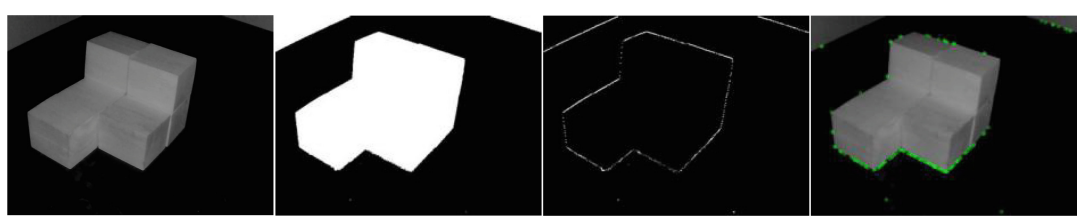

Fig. 13. Edge detection and corner detection pictures after two-dimensional OSTU processing.

As shown in Fig. 13, the two-dimension OSTU algorithm, which is averaged by two-dimensional threshold value, can fully extract the object foreground information in the night vision environment, as shown in Fig. 13, the second picture, and accurately detect the position of edges and corners according to the foreground information, as shown in Fig. 13, the third and fourth pictures.

\section{The Conclusion}

In the study of binocular "five-distance" measurement of hazardous chemicals, aiming at the problem of cargo feature extraction with fuzzy imaging or close gray value of binocular camera in night vision environment, an algorithm of two-dimensional otsu threshold function window design and two-dimensional threshold average is proposed. Experiments show that the algorithm can effectively remove noise and improve the accuracy of edge and corner extraction by combining foreground and background information.

Project Supporter. This paper is supported by the national key research and development project (2016YFC0801500). As well as the Innovation Ability Promotion Project of Municipal Colleges of Beijing Education Commission (2016014222000041).

\section{References}

1. Wenyu, J.: Major events and WeChat opinion - to "Tianjin port on August 12 extra-large fire explosion accident" as an example. China Newspaper Industry, 40-41 (2018)

2. Kai, Z.: How to prepare for the safety of the warehouse tank farm management - think of from zhangjiakou 11.28 accident. Chemical Enterp. Manage., 59-60 (2019)

3. Lichen, S., Rujun, W., Yingquan, D.: Problems and suggestions on safety supervision of dangerous chemicals in China. J. Saf. Sci. Technol., 161-166 (2014)

4. Bo, D., Zhichao, L., Xuejun, L., Xin, L., Guang, Y.: UWB location technology of hazardous chemicals stacking warehouse based on Thiessen polygon. J Chem. Ind. Eng. China, 878884 (2016)

5. Xuejun, L., Yuchen, W., Bixian, Y.: For warehouse and laser ranging in adaptive hierarchical clustering denoising algorithm research. Comput. Sci., 208-211, 217 (2018) 
6. Bing, F., Zhang, W., Jun, S..: Based on quantum particle swarm optimization (PSO) algorithm of Ostu threshold image segmentation. Comput. Eng. Des., 3429-3431, 3434 (2008)

7. Yang, Y., Lin, K., Chuang, Y.: Adaptive particle swarm optimization of two-dimensional image of OSTU threshold segmentation algorithm is proposed. J. Electron. Meas. Instrum., 827-832 (2017)

8. Zhibing, H., Tao, L., Kun, Y.: Based on the improved two-dimensional Ostu algorithm of SAR image segmentation method. J. Naval Aeronaut. Astronaut. Univ., 153-157 (2010)

9. Wei, W., Jin-jin, Z., Hong-jun, Z.: Segmenting, removing and ranking partial blur. SIViP 8(4), 647-655 (2014). https://doi.org/10.1007/s11760-013-0573-8

10. Tao, Z., Dequn, L., Xinnian, W.: Based on texture feature of reference image fuzzy degree evaluation method. Comput. Eng. Appl., 185-191 (2012)

11. Wei, W.: Fuzzy removal and quality evaluation research based on single image. University of Science and Technology of China, 133 (2014) 